Pacific

Journal of

Mathematics

\title{
PRODUCTS OF PARTIAL NORMAL SUBGROUPS
}

\section{ELLEN HENKE}

Volume 279 No. 1-2

December 2015 


\title{
PRODUCTS OF PARTIAL NORMAL SUBGROUPS
}

\author{
ELLEN HENKE
}

In memory of Robert Steinberg.

\begin{abstract}
We show that the product of two partial normal subgroups of a locality (in the sense of Chermak) is again a partial normal subgroup. This generalizes a theorem of Chermak and fits into the context of building a local theory of localities.
\end{abstract}

\section{Introduction}

Localities were introduced by Andrew Chermak [2013], in the context of his proof of the existence and uniqueness of centric linking systems. Roughly speaking, localities are group-like structures which are essentially the "same" as the transporter systems of Oliver and Ventura [2007]; see the appendix to [Chermak 2013]. As centric linking systems are special cases of transporter systems, the existence of centric linking systems implies that there is a locality attached to every fusion system. It is work in progress of Chermak to build a local theory of localities similar to the local theory of fusion systems as developed by Aschbacher [2008; 2011]. In fact, it seems often an advantage to work inside of localities, where some group theoretical concepts and constructions can be expressed more naturally than in fusion systems. Thus, one can hope to improve the local theory of fusion systems, once a way of translating between fusion systems and localities is established. The results of this paper can be considered as first evidence that some constructions are easier in the world of localities. We prove that the product of partial normal subgroups of a locality is itself a partial normal subgroup, whereas in fusion systems the product of normal subsystems has only been defined in special cases; see [Aschbacher 2011, Theorem 3]. It is work in progress of Chermak to show that there is a one-to-one correspondence between the normal subsystems of a saturated fusion system $\mathcal{F}$ and the partial normal subgroups of a linking locality attached to $\mathcal{F}$ in the sense of [Henke 2015, Definition 2]. This is one reason why our result seems particularly important in the case of linking localities. Another reason is that the concept of a linking locality generalizes properties of localities corresponding to centric linking

MSC2010: 20D20, 20N99.

Keywords: fusion systems, localities, transporter systems. 
systems and is thus interesting for studying the homotopy theory of fusion systems; see [Broto et al. 2003; 2005; 2007; Henke 2015]. It is however crucial for our proof that we work with arbitrary localities, since our arguments rely heavily on the theory of quotient localities introduced by Chermak [2015], and a quotient of a linking locality is not necessarily a linking locality again. Thus, we feel that the method of our proof gives evidence for the value of studying localities in general rather than restricting attention only to the special case of linking localities.

To describe the results of this paper in more detail, let $\mathcal{L}$ be a partial group as defined in [Chermak 2013, Definition 2.1; 2015, Definition 1.1]. Thus, there is an involutory bijection $\mathcal{L} \rightarrow \mathcal{L}, f \mapsto f^{-1}$, called an "inversion", and a multivariable product $\Pi$ which is only defined on certain words in $\mathcal{L}$. Let $\boldsymbol{D}$ be the domain of the product; i.e., $\boldsymbol{D}$ is a set of words in $\mathcal{L}$ and $\Pi$ is a map $\boldsymbol{D} \rightarrow \mathcal{L}$. Following Chermak, we call a nonempty subset $\mathcal{H}$ of $\mathcal{L}$ a partial subgroup of $\mathcal{L}$ if $h^{-1} \in \mathcal{H}$ for all $h \in \mathcal{H}$ and $\Pi(v) \in \mathcal{H}$ for all words $v$ in the alphabet $\mathcal{H}$ with $v \in \boldsymbol{D}$. A partial subgroup $\mathcal{N}$ is called a partial normal subgroup if $x^{f}:=\Pi\left(f^{-1}, x, f\right) \in \mathcal{N}$ for all $x \in \mathcal{N}$ and $f \in \mathcal{L}$ for which $\left(f^{-1}, x, f\right) \in \boldsymbol{D}$. Given two subsets $\mathcal{M}$ and $\mathcal{N}$ of $\mathcal{L}$, the product $\mathcal{M N}$ is naturally defined by

$$
\mathcal{M N}=\{\Pi(m, n): m \in \mathcal{M}, n \in \mathcal{N},(m, n) \in \boldsymbol{D}\} .
$$

The problem is however to show that this is again a partial normal subgroup if $\mathcal{M}$ and $\mathcal{N}$ are partial normal subgroups. Indeed, as we show in Example 2.3, this is not true in general if $\mathcal{L}$ is an arbitrary partial group. It is true however in the important case that $(\mathcal{L}, \Delta, S)$ is a locality. Chermak [2015, Theorem 5.1] proved this in a special case and we build on his result to prove the general case. More precisely, we prove the following theorem:

Theorem 1. Let $(\mathcal{L}, \Delta, S)$ be a locality and let $\mathcal{M}, \mathcal{N}$ be partial normal subgroups of $\mathcal{L}$. Then $\mathcal{M N}=\mathcal{N} \mathcal{M}$ is a partial normal subgroup of $\mathcal{L}$ and $(\mathcal{M N}) \cap S=$ $(\mathcal{M} \cap S)(\mathcal{N} \cap S)$. Moreover, for every $g \in \mathcal{M N}$ there exists $m \in \mathcal{M}$ and $n \in \mathcal{N}$ such that $(m, n) \in \boldsymbol{D}, g=\Pi(m, n)$, and $S_{g}=S_{(m, n)}$.

To understand the technical conditions stated in the last sentence of the theorem, we recall from [Chermak 2013; 2015] that

$$
S_{g}=\left\{s \in S:\left(g^{-1}, s, g\right) \in \boldsymbol{D} \text { and } s^{g} \in S\right\}
$$

for any $g \in \mathcal{L}$. Moreover, for a word $v=\left(g_{1}, \ldots, g_{n}\right)$ in $\mathcal{L}, S_{v}$ is the set of all $s \in S$ such that there exist $x_{0}, \ldots, x_{n} \in S$ with $s=x_{0},\left(g_{i}^{-1}, x_{i-1}, g_{i}\right) \in \boldsymbol{D}$ and $x_{i-1}^{g_{i}}=x_{i}$ for $i=1, \ldots, n$. By [op. cit, Proposition 2.6 and Corollary 2.7], the sets $S_{g}$ and $S_{v}$ are subgroups of $S, S_{g} \in \Delta$ for any $g \in \mathcal{L}$, and $S_{v} \in \Delta$ if and only if $v \in D$. Therefore, the condition $S_{g}=S_{(m, n)}$ stated in the theorem is crucial for proving that certain products are defined in $\mathcal{L}$. This is particularly important for the proof of our 
next theorem which concerns products of more than two partial normal subgroups. Given subsets $\mathcal{N}_{1}, \mathcal{N}_{2}, \ldots, \mathcal{N}_{l}$ of $\mathcal{L}$ define their product via

$\mathcal{N}_{1} \mathcal{N}_{2} \cdots \mathcal{N}_{l}:=\left\{\Pi\left(n_{1}, n_{2}, \cdots, n_{l}\right):\left(n_{1}, n_{2}, \ldots, n_{l}\right) \in \boldsymbol{D}, n_{i} \in \mathcal{N}_{i}\right.$ for $\left.1 \leq i \leq l\right\}$.

We prove:

Theorem 2. Let $\mathcal{N}_{1}, \mathcal{N}_{2}, \ldots, \mathcal{N}_{l}$ be partial normal subgroups of a locality $(\mathcal{L}, \Delta, S)$. Then $\mathcal{N}_{1} \mathcal{N}_{2} \cdots \mathcal{N}_{l}$ is a partial normal subgroup of $\mathcal{L}$. Moreover, the following hold:

(1) $\mathcal{N}_{1} \mathcal{N}_{2} \cdots \mathcal{N}_{l}=\left(\mathcal{N}_{1} \cdots \mathcal{N}_{k}\right)\left(\mathcal{N}_{k+1} \cdots \mathcal{N}_{l}\right)$ for every $1 \leq k<l$.

(2) $\mathcal{N}_{1} \mathcal{N}_{2} \cdots \mathcal{N}_{l}=\mathcal{N}_{1 \sigma} \mathcal{N}_{2 \sigma} \cdots \mathcal{N}_{\text {lo }}$ for every permutation $\sigma \in S_{l}$.

(3) For every $g \in \mathcal{N}_{1} \ldots \mathcal{N}_{l}$ there exists $\left(n_{1}, \ldots, n_{l}\right) \in \boldsymbol{D}$ with $n_{i} \in \mathcal{N}_{i}$ for every $i=1, \ldots, l, g=\Pi\left(n_{1}, \ldots, n_{l}\right)$, and $S_{g}=S_{\left(n_{1}, \ldots, n_{l}\right)}$.

As already mentioned above, it is work in progress of Andrew Chermak to show that for every fusion system $\mathcal{F}$ and a linking locality $(\mathcal{L}, \Delta, S)$ attached to $\mathcal{F}$ there is a one-to-one correspondence between the normal subsystems of $\mathcal{F}$ and the partial normal subgroups of $\mathcal{L}$. When this work is complete, our results will imply the existence of a product of an arbitrary finite number of normal subsystems of $\mathcal{F}$.

In this text only relatively few demands will be made on understanding the concepts introduced in [Chermak 2013; 2015]. In Section 2, we point the reader to the few general results needed about partial groups, give a concise definition of a locality and review some basic facts about localities. In Section 3, we summarize what is needed about partial normal subgroups and quotient localities.

\section{Partial groups and localities}

We refer the reader to [Chermak 2013, Definition 2.1] or [Chermak 2015, Definition 1.1] for the precise definition of a partial group, and to the elementary properties of partial groups stated in [2013, Lemma 2.2] or [2015, Lemma 1.4]. Adapting Chermak's notation we write $\boldsymbol{W}(\mathcal{L})$ for the set of words in a set $\mathcal{L}, \varnothing$ for the empty word, and $v_{1} \circ v_{2} \circ \cdots \circ v_{n}$ for the concatenation of words $v_{1}, \ldots, v_{n} \in \boldsymbol{W}(\mathcal{L})$.

For the remainder of this text let $\mathcal{L}$ be a partial group with product $\Pi: D \rightarrow \mathcal{L}$ defined on the domain $D \subseteq W(\mathcal{L})$.

Again following Chermak's notation, we set $\mathbf{1}=\Pi(\varnothing)$. Moreover, given a word $v=\left(f_{1}, \ldots, f_{n}\right) \in \boldsymbol{D}$, we write $f_{1} f_{2} \ldots f_{n}$ for the product $\Pi(v)$. Recall the definitions of partial subgroups and partial normal subgroups from the introduction. Note that a partial subgroup of $\mathcal{L}$ is always a partial group itself whose product is the restriction of the product $\Pi$ to $\boldsymbol{W}(\mathcal{H}) \cap \boldsymbol{D}$. Observe furthermore that $\mathcal{L}$ forms a group in the usual sense if $\boldsymbol{W}(\mathcal{L})=\boldsymbol{D}$; see [op. cit., Lemma 1.3]. So it makes sense 
to call a partial subgroup $\mathcal{H}$ of $\mathcal{L}$ a subgroup of $\mathcal{L}$ if $\boldsymbol{W}(\mathcal{H}) \subseteq \boldsymbol{D}$. In particular, we can talk about $p$-subgroups of $\mathcal{L}$ meaning subgroups of $\mathcal{L}$ whose order is a power of $p$.

We will need the Dedekind lemma [Chermak 2015, Lemma 1.10] in the following slightly more general form:

2.1 (Dedekind lemma). Let $\mathcal{H}, \mathcal{K}, \mathcal{A}$ be subsets of $\mathcal{L}$ such that $\mathcal{A}$ is a partial subgroups of $\mathcal{L}$ and $\mathcal{K} \subseteq \mathcal{A}$. Then $\mathcal{A} \cap(\mathcal{H} \mathcal{K})=(\mathcal{A} \cap \mathcal{H}) \mathcal{K}$ and $\mathcal{A} \cap(\mathcal{K} \mathcal{H})=\mathcal{K}(\mathcal{A} \cap \mathcal{H})$. Proof. Clearly, $(\mathcal{A} \cap \mathcal{H}) \mathcal{K} \subseteq \mathcal{A} \cap(\mathcal{H K})$. Taking $h \in \mathcal{H}$ and $k \in \mathcal{K}$ with $(h, k) \in \boldsymbol{D}$ and $h k \in \mathcal{A}$, we have $\left(h, k, k^{-1}\right) \in \boldsymbol{D}$ by [op. cit, Lemma 1.4(d)] and then $h=$ $h\left(k k^{-1}\right)=(h k) k^{-1} \in \mathcal{A}$ as $\mathcal{K} \subseteq \mathcal{A}$ and $\mathcal{A}$ is a partial subgroup. Hence, $h \in \mathcal{A} \cap \mathcal{H}$ and $h k \in(\mathcal{A} \cap \mathcal{H}) \mathcal{K}$. The second equation follows similarly.

Before we continue with more definitions, we illustrate the concepts we mentioned so far with examples. For this purpose we say that two groups $G_{1}$ and $G_{2}$ form an amalgam, if the set-theoretic intersection $G_{1} \cap G_{2}$ is a subgroup of both $G_{1}$ and $G_{2}$, and the restriction of the multiplication on $G_{1}$ to a multiplication on $G_{1} \cap G_{2}$ is the same as the restriction of the multiplication on $G_{2}$ to a multiplication on $G_{1} \cap G_{2}$.

Example 2.2. Let $G_{1}$ and $G_{2}$ be groups which form an amalgam. Set $\mathcal{L}=G_{1} \cup G_{2}$ and $\boldsymbol{D}=\boldsymbol{W}\left(G_{1}\right) \cup \boldsymbol{W}\left(G_{2}\right)$. Define a partial product $\Pi: \boldsymbol{D} \rightarrow \mathcal{L}$ by sending $v=\left(f_{1}, \ldots, f_{n}\right) \in \boldsymbol{W}\left(G_{i}\right)$ to the product $f_{1} \ldots f_{n}$ in the group $G_{i}$ for $i=1,2$. Define an inversion $\mathcal{L} \rightarrow \mathcal{L}$ by sending $f \in G_{i}$ to the inverse of $f$ in the group $G_{i}$ for $i=1,2$. Then $\mathcal{L}$ with these structures forms a partial group. (For readers familiar with the concept of an objective partial group as introduced in [Chermak 2013, Definition 2.6] or [Chermak 2015, Definition 2.1] we mention that, setting $\Delta:=\left\{G_{1}, G_{2}\right\},(\mathcal{L}, \Delta)$ is an objective partial group if $G_{1} \cap G_{2}$ is properly contained in $G_{1}$ and $G_{2}$.)

Let $\mathcal{K}$ be a subset of $\mathcal{L}$. Then $\mathcal{K}$ is a partial subgroup of $\mathcal{L}$ if and only if $\mathcal{K} \cap G_{i}$ is a subgroup of $G_{i}$ for each $i=1,2$. The subset $\mathcal{K}$ is a subgroup of $\mathcal{L}$ if and only if $\mathcal{K}$ is a subgroup of $G_{i}$ for some $i=1,2$. Moreover, $\mathcal{K}$ is a partial normal subgroup of $\mathcal{L}$ if and only if $\left(\mathcal{K} \cap G_{i}\right) \unlhd G_{i}$ for $i=1,2$.

We use the construction method introduced in the previous example to show that the product of two partial normal subgroups of a partial group is not in general itself a partial normal subgroup.

Example 2.3. Let $G_{1} \cong C_{2} \times C_{4}$ and let $G_{2}$ be a dihedral group of order 16. Choose $G_{1}$ and $G_{2}$ such that $G_{1}$ and $G_{2}$ form an amalgam with $G_{1} \cap G_{2} \cong C_{2} \times C_{2}$ and $\Phi\left(G_{1}\right)=Z\left(G_{2}\right)$. Let $\mathcal{M}$ and $\mathcal{N}$ be the two cyclic subgroups of $G_{1}$ of order 4 . Form the locality $\mathcal{L}$ as in Example 2.2. As $G_{1}$ is abelian, a subgroup $\mathcal{K}$ of $G_{1}$ is normal in $G_{1}$ and thus a partial normal subgroup of $\mathcal{L}$ if and only if $\mathcal{K} \cap G_{2} \unlhd G_{2}$. As $G_{1} \cap G_{2} \cong C_{2} \times C_{2}$ and $\mathcal{M}$ and $\mathcal{N}$ are cyclic of order 4 , we have $\mathcal{M} \cap G_{2}=$ 
$\mathcal{N} \cap G_{2}=\Phi\left(G_{1}\right)=Z\left(G_{2}\right) \unlhd G_{2}$. Thus $\mathcal{M}$ and $\mathcal{N}$ are partial normal subgroups of $\mathcal{L}$. The product $\mathcal{M N}$ in $\mathcal{L}$ is the same as the product $\mathcal{M N}$ in $G_{1}$ and thus equal to $G_{1}$. However, as $G_{2}$ does not have a normal fours subgroup, $G_{1} \cap G_{2}$ is not normal in $G_{2}$ and thus $\mathcal{M N}=G_{1}$ is not a partial normal subgroup of $\mathcal{L}$.

The previous example shows that the concept of a partial group (and even the concept of an objective partial group) is too general for our purposes. Therefore, we will focus on localities. We give a definition of a locality which, in contrast to the definition given by Chermak [2013; 2015], does not require the reader to be familiar with the definition of an objective partial group and can easily seen to be equivalent to Chermak's definition. For any $g \in \mathcal{L}, \boldsymbol{D}(g)$ denotes the set of $x \in \mathcal{L}$ with $\left(g^{-1}, x, g\right) \in \boldsymbol{D}$. Thus, $\boldsymbol{D}(g)$ denotes the set of elements $x \in \mathcal{L}$ for which the conjugation $x^{g}:=\Pi\left(g^{-1}, x, g\right)$ is defined. If $g \in \mathcal{L}$ and $X \subseteq \boldsymbol{D}(g)$ we set $X^{g}:=\left\{x^{g}: x \in X\right\}$. If we write $X^{g}$ for some $g \in \mathcal{L}$ and some subset $X \subseteq \mathcal{L}$, we will always implicitly mean that $X \subseteq \boldsymbol{D}(g)$.

Definition 2.4. We say that $(\mathcal{L}, \Delta, S)$ is a locality if the partial group $\mathcal{L}$ is finite as a set, $S$ is a $p$-subgroup of $\mathcal{L}, \Delta$ is a nonempty set of subgroups of $S$, and the following conditions hold:

(L1) $S$ is maximal with respect to inclusion among the $p$-subgroups of $\mathcal{L}$.

(L2) A word $\left(f_{1}, \ldots, f_{n}\right) \in \boldsymbol{W}(\mathcal{L})$ is an element of $\boldsymbol{D}$ if and only if there exist $P_{0}, \ldots, P_{n} \in \Delta$ such that

$$
P_{i-1} \subseteq \boldsymbol{D}\left(f_{i}\right) \text { and } P_{i-1}^{f_{i}}=P_{i} .
$$

(L3) For any subgroup $Q$ of $S$, for which there exist $P \in \Delta$ and $g \in \mathcal{L}$ with $P \subseteq \boldsymbol{D}(g)$ and $P^{g} \leq Q$, we have $Q \in \Delta$.

If $(\mathcal{L}, \Delta, S)$ is a locality and $v=\left(f_{1}, \ldots, f_{n}\right) \in \boldsymbol{W}(\mathcal{L})$, then we say that $v \in \boldsymbol{D}$ via $P_{0}, \ldots, P_{n}$ (or $v \in \boldsymbol{D}$ via $P_{0}$ ), if $P_{0}, \ldots, P_{n} \in \Delta$ and $(*)$ holds.

From now on let $(\mathcal{L}, \Delta, S)$ be a locality.

Note that $P=P^{1} \leq S$ for all $P \in \Delta$. As $\Delta \neq \varnothing$, property (L3) implies thus $S \in \Delta$. For any $g \in \mathcal{L}$, write $c_{g}$ for the conjugation map

$$
c_{g}: \boldsymbol{D}(g) \rightarrow \mathcal{L}, x \mapsto x^{g} .
$$

Recall the definitions of $S_{g}$ and $S_{v}$ from the introduction. Note that $S_{g} \subseteq \boldsymbol{D}(g)$. For any subgroup $X$ of $\mathcal{L}$ set

$$
N_{\mathcal{L}}(X):=\left\{f \in \mathcal{L}: X \subseteq \boldsymbol{D}(f), X^{f}=X\right\} .
$$

2.5 (Important properties of localities). The following hold:

(a) $N_{\mathcal{L}}(P)$ is a subgroup of $\mathcal{L}$ for each $P \in \Delta$. 
(b) Let $P \in \Delta$ and $g \in \mathcal{L}$ with $P \subseteq S_{g}$. Then $Q:=P^{g} \in \Delta$ (so in particular $Q$ is a subgroup of $S)$. Moreover, $N_{\mathcal{L}}(P) \subseteq \boldsymbol{D}(g)$ and

$$
c_{g}: N_{\mathcal{L}}(P) \rightarrow N_{\mathcal{L}}(Q)
$$

is an isomorphism of groups.

(c) Let $w=\left(g_{1}, \ldots, g_{n}\right) \in \boldsymbol{D}$ via $\left(X_{0}, \ldots, X_{n}\right)$. Then

$$
c_{g_{1}} \circ \cdots \circ c_{g_{n}}=c_{\Pi(w)}
$$

is a group isomorphism $N_{\mathcal{L}}\left(X_{0}\right) \rightarrow N_{\mathcal{L}}\left(X_{n}\right)$.

(d) For every $g \in \mathcal{L}, S_{g} \in \Delta$. In particular, $S_{g}$ is a subgroup of $S$.

(e) For any $w \in \boldsymbol{W}(\mathcal{L}), S_{w}$ is a subgroup of $S_{\Pi(w)}$, and $S_{w} \in \Delta$ if and only if $w \in \boldsymbol{D} .{ }^{1}$

Proof. Properties (a)-(c) correspond to statements in [Chermak 2015, Lemma 2.3] except for the fact stated in (b) that $Q \in \Delta$. This is however true by [op. cit., Proposition 2.6(c)]. Property (d) is true by [op. cit., Proposition 2.6(a)] and property (e) is stated in [op. cit., Corollary 2.7].

\section{Partial normal subgroups and quotient localities}

In this section we continue to assume that $(\mathcal{L}, \Delta, S)$ is a locality. The following theorem is a special case of Theorem 1 and will be used to prove the more general theorem.

Theorem 3.1. Let $\mathcal{M}, \mathcal{N}$ be partial normal subgroups of $\mathcal{L}$ such that $\mathcal{M} \cap \mathcal{N}=1$. Then $\mathcal{M N}=\mathcal{N} \mathcal{M}$ is a partial normal subgroup of $\mathcal{L}$. Moreover, for any $f \in \mathcal{M N}$ there exists $m \in \mathcal{M}$ and $n \in \mathcal{N}$ such that $(m, n) \in \boldsymbol{D}, f=m n$, and $S_{f}=S_{(m, n)}$.

Proof. As $\mathcal{M} \cap \mathcal{N} \subseteq S$, it follows from [Chermak 2015, Lemma 5.3] that $\mathcal{M}$ normalizes $\mathcal{N} \cap S$ and $\mathcal{N}$ normalizes $\mathcal{M} \cap S$. So by [op. cit., Theorem 5.1], $\mathcal{M N}=\mathcal{N} \mathcal{M}$ is a partial normal subgroup of $\mathcal{L}$. Moreover, by [op. cit., Lemma 5.2], for any $f \in \mathcal{M N}$ there exist $m \in \mathcal{M}$ and $n \in \mathcal{N}$ such that $(m, n) \in \boldsymbol{D}, f=m n$, and $S_{f}=S_{(m, n)}$.

To deduce Theorem 1 from Theorem 3.1, we need the theory of quotient localities developed in [Chermak 2015]; see also [Chermak 2013, Sections 3 and 4]. For the convenience of the reader we quickly summarize this theory here. After that we state some more specialized lemmas needed in our proof.

Throughout let $\mathcal{K}$ be a partial normal subgroup of $\mathcal{L}$ and $T=S \cap \mathcal{K}$.

3.2. (a) $T$ is strongly closed in $(\mathcal{L}, \Delta, S)$; that is, $t^{g} \in T$ for every $g \in \mathcal{L}$ and every $t \in T \cap S_{g}$. In particular, $T^{g}=T$ for any $g \in \mathcal{L}$ with $T \subseteq S_{g}$.

(b) $T$ is maximal in the poset of p-subgroups of $\mathcal{N}$.

\footnotetext{
${ }^{1}$ Recall the definition of $S_{\left(g_{1}, \ldots, g_{n}\right)}$ from the introduction.
} 
Proof. Let $g \in \mathcal{L}$ and $t \in T \cap S_{g}$. Then $t^{g} \in S$ and, as $\mathcal{N}$ is a partial normal subgroup, $t^{g} \in \mathcal{N}$. Hence, $t^{g} \in S \cap \mathcal{N}=T$. This proves (a). Property (b) is proved in [Chermak 2015, Lemma 3.1(c)].

We write $\uparrow_{\mathcal{K}}$ for the relation $\uparrow$ introduced in [op. cit., Definition 3.6], but with the partial normal subgroup $\mathcal{N}$ replaced by $\mathcal{K}$. Thus $\uparrow_{\mathcal{K}}$ is a relation on the set $\mathcal{L} \circ \Delta$ of pairs $(f, P) \in \mathcal{L} \times \Delta$ with $P \leq S_{f}$. For $(f, P),(g, Q) \in \mathcal{L} \circ \Delta$, we have $(f, P) \uparrow_{\mathcal{K}}(g, Q)$ if there exist $x \in N_{\mathcal{K}}(P, Q)$ and $y \in N_{\mathcal{K}}\left(P^{f}, Q^{g}\right)$ such that $x g=f y$. We say then $(f, P) \uparrow_{\mathcal{K}}(g, Q)$ via $(x, y)$. One easily sees that $\uparrow_{\mathcal{K}}$ is reflexive and transitive. Moreover, $(f, P) \uparrow_{\mathcal{K}}\left(f, S_{f}\right)$ via $(\mathbf{1}, \mathbf{1})$. An element $f \in \mathcal{L}$ is called $\uparrow_{\mathcal{K}}$-maximal if $\left(f, S_{f}\right)$ is maximal with respect to the relation $\uparrow_{\mathcal{K}}$ (i.e., if $\left(f, S_{f}\right) \uparrow_{\mathcal{K}}(g, Q)$ implies $(g, Q) \uparrow_{\mathcal{K}}\left(f, S_{f}\right)$ for any $\left.(g, Q) \in \mathcal{L} \circ \Delta\right)$. We summarize some important technical properties of the relation $\uparrow_{\mathcal{K}}$ in the following lemma.

3.3. The following hold:

(a) Every element of $N_{\mathcal{L}}(S)$ is $\uparrow \mathcal{K}$-maximal. In particular, every element of $S$ is $\uparrow \mathcal{K}$-maximal.

(b) If $f \in \mathcal{L}$ is $\uparrow \mathcal{K}$-maximal, then $T \leq S_{f}$.

(c) (Stellmacher's splitting lemma) Let $(x, f) \in \boldsymbol{D}$ such that $x \in \mathcal{K}$ and $f$ is $\uparrow \mathcal{K}$-maximal. Then $S_{(x, f)}=S_{x f}$.

Proof. Property (a) is [Chermak 2015, Lemma 3.7(a)], (b) is [op. cit., Proposition 3.9], and (c) is [op. cit., Lemma 3.12].

The relation $\uparrow_{\mathcal{K}}$ is crucial for defining a quotient locality $\mathcal{L} / \mathcal{K}$ somewhat analogously to quotients of groups. A coset of $\mathcal{K}$ in $\mathcal{L}$ is of the form

$$
\mathcal{K} f=\{k f: k \in \mathcal{K},(k, f) \in \boldsymbol{D}\}
$$

for some $f \in \mathcal{L}$. A maximal coset of $\mathcal{K}$ is a coset which is maximal with respect to inclusion among the cosets of $\mathcal{K}$ in $\mathcal{L}$. The set of these maximal cosets is denoted by $\mathcal{L} / K$.

3.4. The following hold:

(a) $f \in \mathcal{L}$ is $\uparrow \mathcal{K}$-maximal if and only if $\mathcal{K} f$ is a maximal coset.

(b) The maximal cosets of $\mathcal{K}$ form a partition of $\mathcal{L}$.

Proof. This is [op. cit., Proposition 3.14(b),(c),(d)].

The reader might note that what we call a coset would be more precisely called a right coset. The distinction does however not matter very much, since we are mostly interested in the maximal cosets and, by [op. cit., Proposition 3.14(a)], we 
have $\mathcal{K} f=f \mathcal{K}$ for any $\uparrow \mathcal{K}$-maximal element $f \in \mathcal{L}$. By 3.4(b), we can define a map

$$
\rho: \mathcal{L} \rightarrow \overline{\mathcal{L}}:=\mathcal{L} / \mathcal{K}
$$

sending $f \in \mathcal{L}$ to the unique maximal coset of $\mathcal{K}$ containing $f$. This should be thought of as a "quotient map". We adopt the bar notation similarly as used for groups. Thus, if $X$ is an element or a subset of $\mathcal{L}$, then $\bar{X}$ denotes the image of $X$ under $\rho$. Furthermore, if $X$ is an element or a subset of $W(\mathcal{L})$ then $\bar{X}$ denotes the image of $X$ under $\rho^{*}$, where $\rho^{*}$ denotes the map $\boldsymbol{W}(\mathcal{L}) \rightarrow \boldsymbol{W}(\mathcal{L})$ with $\left(f_{1}, \ldots, f_{n}\right) \rho^{*}=\left(f_{1} \rho, \ldots, f_{n} \rho\right)$. In particular,

$$
\overline{\boldsymbol{D}}=\boldsymbol{D} \rho^{*} .
$$

We note:

3.5. Let $f, g \in \mathcal{L}$ such that $\bar{g}=\bar{f}$ and $f$ is $\uparrow \mathcal{K}$-maximal. Then $g \in \mathcal{K} f$.

Proof. By $3.4($ a), $\mathcal{K} f$ is a maximal coset, so $\bar{g}=\bar{f}=\mathcal{K} f$ by the definition of $\rho$. Hence, again by the definition of $\rho, g \in \mathcal{K} f$.

Recall the definition of a homomorphism of a partial groups from [Chermak 2013, Definition 3.1] and [Chermak 2015, Definition 1.11]. By [op. cit., Lemma 3.16], there is a unique mapping $\bar{\Pi}: \overline{\boldsymbol{D}} \rightarrow \overline{\mathcal{L}}$ and a unique involutory bijection $\bar{f} \mapsto \bar{f}^{-1}$ such that $\overline{\mathcal{L}}$ with these structures is a partial group and $\rho$ is a homomorphism of partial groups. Since $\rho$ is a homomorphism, we have $\bar{\Pi}(\bar{v})=\bar{\Pi}\left(v \rho^{*}\right)=\Pi(v) \rho=$ $\overline{\Pi(v)}$ for $v \in \boldsymbol{D}$ and $\bar{f}^{-1}=\overline{f^{-1}}$ by the definition of a homomorphism of partial groups and by [op. cit., Lemma 1.13]. In particular, $\overline{\mathbf{1}}=\bar{\Pi}(\varnothing)$ is the identity element in $\overline{\mathcal{L}}$. So $\rho$ has kernel $\operatorname{ker}(\rho)=\{f \in \mathcal{L}: \bar{f}=\overline{\mathbf{1}}\}=\mathcal{K} \mathbf{1}=\mathcal{K}$. By [op. cit., Proposition 4.2], $(\overline{\mathcal{L}}, \bar{\Delta}, \bar{S})$ is a locality for $\bar{\Delta}:=\{\bar{P}: P \in \Delta\}$. We will use this important fact throughout without further reference. We remark:

3.6. Let $v=\left(f_{1}, \ldots, f_{n}\right) \in \boldsymbol{W}(\mathcal{L})$ such that each $f_{i}$ is $\uparrow_{\mathcal{K}}$-maximal and $\bar{v} \in \overline{\boldsymbol{D}}$. Then $v \in \boldsymbol{D}$ and $\bar{\Pi}(\bar{v})=\overline{\Pi(v)}$.

Proof. As $\bar{v} \in \overline{\boldsymbol{D}}$, there is $u=\left(g_{1}, \ldots, g_{n}\right) \in \boldsymbol{D}$ such that $\bar{u}=\bar{v}$. Then $\overline{g_{i}}=\overline{f_{i}}$ for $i=1, \ldots, n$, i.e., $g_{i} \in \mathcal{K} f_{i}$ by 3.5. Now by [op. cit., Proposition 3.14(e)], $v \in \boldsymbol{D}$. As seen above, since $\rho$ is a homomorphism of partial groups, $\bar{\Pi}(\bar{v})=\overline{\Pi(v)}$.

There is a nice correspondence between the partial subgroups of $\mathcal{L}$ containing $\mathcal{K}$ and the partial subgroups of $\overline{\mathcal{L}}$.

3.7. Let $\mathfrak{H}$ be the set of partial subgroups of $\mathcal{L}$ containing $\mathcal{K}$.

(a) Let $\mathcal{H} \in \mathfrak{H}$. Then the maximal cosets of $\mathcal{K}$ contained in $\mathcal{H}$ form a partition of $\mathcal{H}$.

(b) Write $\overline{\mathfrak{H}}$ for the set of partial subgroups of $\mathcal{L}$. Then the map $\mathfrak{H} \rightarrow \overline{\mathfrak{H}}$ with $\mathcal{H} \mapsto \overline{\mathcal{H}}$ is well defined and a bijection. Moreover, for any $\mathcal{H} \in \mathfrak{H}$, we have $\overline{\mathcal{H}} \unlhd \overline{\mathcal{L}}$ if and only if $\mathcal{H} \unlhd \mathcal{L}$. 
Proof. Property (a) is [Chermak 2015, Lemma 3.15]. The map $\rho$ is a homomorphism of partial groups and $(\overline{\mathcal{L}}, \bar{\Delta}, \bar{S})$ is a locality. From the way $\overline{\boldsymbol{D}}$ and $\bar{\Delta}$ are defined, it follows that $\rho$ is a projection in the sense of [op. cit., Definition 4.5]. Hence, property (b) is a reformulation of [op. cit., Proposition 4.8].

3.8. For any subset $X$ of $\mathcal{L}$ and for any partial subgroup $\mathcal{H}$ of $\mathcal{L}$ containing $\mathcal{K}$, $\bar{X} \cap \overline{\mathcal{H}}=\overline{X \cap \mathcal{H}}$.

Proof. Clearly, $\overline{X \cap \mathcal{H}} \subseteq \bar{X} \cap \overline{\mathcal{H}}$. Let now $x \in X$ such that $\bar{x} \in \overline{\mathcal{H}}$. Then there exists $h \in \mathcal{H}$ such that $\bar{x}=\bar{h}$ and, by 3.7(a), we may choose $h$ such that $\mathcal{K} h$ is a maximal coset. By the definition of $\rho$, this means $x \in \mathcal{K} h \subseteq \mathcal{H}$ and hence $x \in X \cap \mathcal{H}$. Thus $\bar{x} \in \overline{X \cap \mathcal{H}}$, proving $\bar{X} \cap \overline{\mathcal{H}} \subseteq \overline{X \cap \mathcal{H}}$.

3.9. Let $R \leq S$. Then $\{f \in \mathcal{L}: \bar{f} \in \bar{R}\}=\mathcal{K} R$.

Proof. Clearly, $\bar{f} \in \bar{R}$ for any $f \in \mathcal{K} R$, as $\mathcal{K}$ is the kernel of $\rho$. Let now $f \in \mathcal{L}$ and $r \in R$ with $\bar{f}=\bar{r}$. As every element of $S$ is $\uparrow \mathcal{K}$-maximal by 3.3(a), it follows from 3.5 that $f \in \mathcal{K} r \subseteq \mathcal{K} R$. This proves the assertion.

3.10. Let $T \leq R \leq S$. Then $R=\{s \in S: \bar{s} \in \bar{R}\}$ and $N_{\bar{S}}(\bar{R})=\overline{N_{S}(R)}$.

Proof. By 3.9 and the Dedekind lemma (2.1), we have $\{s \in S: \bar{s} \in \bar{R}\}=S \cap(\mathcal{K} R)=$ $(S \cap \mathcal{K}) R=T R=R$. Moreover, for any element $t \in S$ with $\bar{t} \in N_{\bar{S}}(\bar{R})$ and any $r \in R$, we have $\bar{r}^{t}=\bar{r}^{\bar{t}} \in \bar{R}$, so $r^{t} \in\{s \in S: \bar{s} \in \bar{R}\}=R$. Hence, $N_{\bar{S}}(\bar{R}) \leq \overline{N_{S}(R)}$. As $\rho$ is a homomorphism of partial groups, $\overline{N_{S}(R)} \subseteq N_{\bar{S}}(\bar{R})$, so the assertion holds.

3.11. For every $f \in \mathcal{L}$ such that $f$ is $\uparrow \mathcal{K}$-maximal, we have ${\overline{S_{f}}}={\overline{S_{\bar{f}}}}_{\overline{\mathrm{f}}}$

Proof. Set $P=S_{f}$ and $Q=P^{f}$. As $\rho$ is a homomorphism of partial groups, one easily observes that $\bar{P} \subseteq \bar{S}_{\bar{f}}$. As $(\overline{\mathcal{L}}, \bar{\Delta}, \bar{S})$ is a locality, $\bar{S}_{\bar{f}}$ is a $p$-group. So assuming the assertion is wrong, there exists $a \in S$ such that $\bar{a} \in N_{\bar{S}_{\bar{f}}}(\bar{P}) \backslash \bar{P}$. As $f$ is $\uparrow$-maximal, $T \leq P=S_{f}$ by 3.3(b). Hence, by 3.10 applied with $P$ in the role of $R, \bar{a} \in \overline{N_{S}(P)}$. So by 3.10 now applied with $N_{S}(P)$ in the role of $R$, $a \in N_{S}(P)$. Using 2.5(a),(b), we conclude that $A:=P\langle a\rangle$ is a $p$-subgroup of the group $N_{\mathcal{L}}(P)$ and that $A^{f}$ is a $p$-subgroup of the group $N_{\mathcal{L}}(Q)$. As $\bar{A} \bar{f} \subseteq \bar{S}$, we have $\bar{A}^{\bar{f}} \subseteq N_{\bar{S}}(\bar{Q})$. By 3.2(a), $T=T^{f} \leq Q$. Thus, by 3.10, $\bar{A}^{\bar{f}} \subseteq \overline{N_{S}(Q)}$. Now 3.9 yields $A^{f} \subseteq\left(\mathcal{K} N_{S}(Q)\right) \cap N_{\mathcal{L}}(Q)=N_{\mathcal{K}}(Q) N_{S}(Q)$, where the last equality uses the Dedekind lemma (2.1). Recall that $N_{\mathcal{L}}(Q)$ is a finite group. Clearly, $N_{\mathcal{K}}(Q)$ is a normal subgroup of $N_{\mathcal{L}}(Q)$. It follows from 3.2(b) that $T \in \operatorname{Syl}_{p}\left(N_{\mathcal{K}}(Q)\right)$. So $N_{S}(Q) \in \operatorname{Syl}_{p}\left(N_{\mathcal{K}}(Q) N_{S}(Q)\right)$ and by Sylow's theorem, there exists $c \in N_{\mathcal{K}}(Q)$ such that $A^{f c} \leq N_{S}(Q)$. Then $(f, P) \uparrow_{\mathcal{K}}(f c, A)$ via $(\mathbf{1}, c)$ contrary to $f$ being $\uparrow \mathcal{K}$-maximal.

3.12. Suppose that $f, g \in \mathcal{L}$ such that $\bar{f}=\bar{g}, S_{f}=S_{g}$, and $f$ is $\uparrow \mathcal{K}$-maximal. Then $g$ is $\uparrow \mathcal{K}$-maximal and $\mathcal{K} f=\mathcal{K} g$. 
Proof. As $f$ is $\uparrow \mathcal{K}$-maximal and $\bar{f}=\bar{g}$, we have $g \in \mathcal{K} f$ by 3.5, i.e., there exists $k \in \mathcal{K}$ with $(k, f) \in \boldsymbol{D}$ and $g=k f$. By Stellmacher's splitting lemma 3.3(c), we have $S_{g}=S_{k f}=S_{(k, f)}$. Hence, $S_{f}=S_{g}=S_{(k, f)}$ and thus $k \in N_{\mathcal{L}}\left(S_{f}\right)$. By 2.5(c), $k^{-1} \in N_{\mathcal{L}}\left(S_{f}\right)$ and $\left(k^{-1}, k, f\right) \in \boldsymbol{D}$ as via $S_{f}$. Hence, $\left(k^{-1}, g\right)=\left(k^{-1}, k f\right) \in \boldsymbol{D}$, $k^{-1} g=k^{-1}(k f)=k^{-1} k f=\left(k^{-1} k\right) f=f$ and $S_{f}^{g}=S_{f}^{f}$. This shows that $\left(f, S_{f}\right) \uparrow \mathcal{K}$ $\left(g, S_{f}\right)$ via $\left(k^{-1}, \mathbf{1}\right)$. We conclude that $g$ is $\uparrow_{\mathcal{K}}$-maximal as $f$ is $\uparrow_{\mathcal{K}}$-maximal and $\uparrow_{\mathcal{K}}$ is transitive. By 3.4, $\mathcal{K} g$ and $\mathcal{K} f$ are both maximal cosets, and the maximal cosets of $\mathcal{K}$ form a partition of $\mathcal{L}$. So it follows that $\mathcal{K} f=\mathcal{K} g$.

\section{Proof of Theorem 1}

Throughout this section assume the hypothesis of Theorem 1. Set

$$
\mathcal{K}:=\mathcal{M} \cap \mathcal{N} .
$$

Observe that $\mathcal{K}$ is a partial normal subgroup of $\mathcal{L}$. As in Section 3, let

$$
\rho: \mathcal{L} \rightarrow \overline{\mathcal{L}}:=\mathcal{L} / \mathcal{K}
$$

be the quotient map sending $f \in \mathcal{L}$ to the unique maximal coset of $\mathcal{K}$ containing $f$, and use the bar notation as introduced there. Set

$$
T:=\mathcal{K} \cap S .
$$

4.1. $\overline{\mathcal{M}} \cap \overline{\mathcal{N}}=1$.

Proof. As $\mathcal{K}$ is contained in $\mathcal{M}$ and $\mathcal{N}$, this is a special case of 3.8.

4.2. We have $\overline{\mathcal{M}} \overline{\mathcal{N}}=\overline{\mathcal{N}} \overline{\mathcal{M}}$, and $\overline{\mathcal{M}} \overline{\mathcal{N}}$ is a partial normal subgroup of $\overline{\mathcal{L}}$. Moreover, for any $x \in \overline{\mathcal{M}} \overline{\mathcal{N}}$, there exist $\bar{m} \in \overline{\mathcal{M}}$ and $\bar{n} \in \overline{\mathcal{N}}$ such that $(\bar{m}, \bar{n}) \in \overline{\boldsymbol{D}}, x=\bar{m} \bar{n}$ and $\bar{S}_{x}=\bar{S}_{(\bar{m}, \bar{n})}$.

Proof. By $3.7($ b), $\overline{\mathcal{M}}$ and $\overline{\mathcal{N}}$ are partial normal subgroups of $\overline{\mathcal{L}}$. By $4.1, \overline{\mathcal{M}} \cap \overline{\mathcal{N}}=1$. Hence, the assertion follows from Theorem 3.1.

4.3. Let $x \in \overline{\mathcal{M}} \overline{\mathcal{N}}$. Then there exist $m \in \mathcal{M}$ and $n \in \mathcal{N}$ with $(m, n) \in \boldsymbol{D}$ such that $m, n$, and $m n$ are $\uparrow \mathcal{K}$-maximal, $x=\bar{m} \bar{n}=\overline{m n}$ and $S_{m n}=S_{(m, n)}$.

Proof. By 4.2, there exist $\bar{m} \in \overline{\mathcal{M}}, \bar{n} \in \overline{\mathcal{N}}$ such that $(\bar{m}, \bar{n}) \in \overline{\boldsymbol{D}}, x=\bar{m} \bar{n}$, and $\bar{S}_{x}=$ $\bar{S}_{(\bar{m}, \bar{n})}$. By 3.7(a), we may furthermore choose preimages $m \in \mathcal{M}$ and $n \in \mathcal{N}$ of $\bar{m}$ and $\bar{n}$ such that $m$ and $n$ are $\uparrow \mathcal{K}$-maximal. Then, by 3.2(a) and 3.3(b), $m, n \in N_{\mathcal{L}}(T)$. By 3.6, $(m, n) \in \boldsymbol{D}$ and $\bar{m} \bar{n}=\overline{m n}$. It remains to prove that $S_{m n}=S_{(m, n)}$ and that $m n$ is $\uparrow \mathcal{K}$-maximal. As an intermediate step we prove the following two properties: $S_{f} \subseteq S_{(m, n)}$ for every $f \in \mathcal{L}$ with $\bar{f}=x$.

$$
S_{f}=S_{(m, n)} \text { for every } \uparrow \mathcal{K} \text {-maximal element } f \in \mathcal{L} \text { with } \bar{f}=x \text {. }
$$


For the proof of (4-1) and (4-2) note first that, by 3.11, $\bar{S}_{\bar{m}}=\overline{S_{m}}$ and $\bar{S}_{\bar{n}}=\overline{S_{n}}$ as $m$ and $n$ are $\uparrow \mathcal{K}$-maximal. Hence,

$$
\bar{S}_{x}=\bar{S}_{(\bar{m}, \bar{n})}=\left\{\bar{s}: \bar{s} \in \bar{S}_{\bar{m}}, \bar{s}^{\bar{m}} \in \bar{S}_{\bar{n}}\right\}=\left\{\bar{s}: s \in S_{m}, \bar{s}^{\bar{m}} \in \bar{S}_{n}\right\} .
$$

If $s \in S_{m}$ then, by definition of $S_{m},\left(m^{-1}, s, m\right) \in \boldsymbol{D}$ and $s^{m} \in S$. Moreover, as $\rho$ is a homomorphism of partial groups, $\overline{s^{m}}=\bar{s}^{\bar{m}}$. So $\bar{s}^{\bar{m}} \in \overline{S_{n}}$ is equivalent to $s^{m} \in S_{n}$ by 3.10 since $T \leq S_{n}$. Hence,

$$
\bar{S}_{x}=\left\{\bar{s}: s \in S_{m}, s^{m} \in S_{n}\right\}=\overline{S_{(m, n)}} .
$$

As $m, n \in N_{\mathcal{L}}(T), T \leq S_{(m, n)}$. Clearly, $\overline{S_{f}} \subseteq \bar{S}_{x}$ for every $f \in \mathcal{L}$ with $\bar{f}=x$. If such $f$ is in addition $\uparrow \mathcal{K}$-maximal, then $\overline{S_{f}}=\bar{S}_{x}$ and $T \leq S_{f}$ by 3.11 and 3.3(b). Now (4-1) and (4-2) follow from 3.10. As $\overline{m n}=\bar{m} \bar{n}=x$, (4-1) yields in particular $S_{m n} \subseteq S_{(m, n)}$ and thus $S_{m n}=S_{(m, n)}$ by 2.5(e). Choosing $f \in \mathcal{L}$ to be $\uparrow \mathcal{K}$-maximal with $\bar{f}=x$, we obtain from (4-2) that $S_{f}=S_{(m, n)}=S_{m n}$. So $m n$ is $\uparrow \mathcal{K}$-maximal by 3.12 completing the proof.

4.4. Let $f \in \mathcal{L}$ with $\bar{f} \in \overline{\mathcal{M}} \overline{\mathcal{N}}$. Then $f \in \mathcal{M N}$ and there exist $m \in \mathcal{M}, n \in \mathcal{N}$ with $(m, n) \in \boldsymbol{D}, f=m n$, and $S_{f}=S_{(m, n)}$.

Proof. By 4.3, we can choose $m \in \mathcal{M}$ and $n \in \mathcal{N}$ with $(m, n) \in \boldsymbol{D}$ such that $m n$ is $\uparrow \mathcal{K}_{\text {-maximal, }} \bar{f}=\overline{m n}$ and $S_{m n}=S_{(m, n)}$. Then there exists $k \in \mathcal{K}$ with $(k, m n) \in \boldsymbol{D}$ and $f=k(m n)$. As $S_{m n}=S_{(m, n)}$, it follows that $S_{(k, m n)}=S_{(k, m, n)}$ and $(k, m, n) \in \boldsymbol{D}$ by $2.5(\mathrm{e})$. Hence, $(\mathrm{km}, n) \in \boldsymbol{D}$ and $f=(\mathrm{km}) n$ by the axioms of a partial group. As $\mathcal{K} \subseteq \mathcal{M}$, we have $k m \in \mathcal{M}$ and so $f=(\mathrm{km}) n \in \mathcal{M N}$. It is now sufficient to show that $S_{(k m, n)}=S_{f}$. As $m n$ is $\uparrow \mathcal{K}$-maximal, it follows from Stellmacher's splitting lemma 3.3(c) that $S_{f}=S_{k(m n)}=S_{(k, m n)}=S_{(k, m, n)} \subseteq S_{(k m, n)}$. By 2.5(e), $S_{(k m, n)} \subseteq S_{(k m) n}=S_{f}$. So $S_{f}=S_{(k m, n)}$, proving the assertion.

Proof of Theorem 1. By 4.2 and 3.7(b), there exists a partial normal subgroup $\mathcal{H}$ of $\mathcal{L}$ containing $\mathcal{K}$ such that $\overline{\mathcal{H}}=\overline{\mathcal{M}} \overline{\mathcal{N}}=\overline{\mathcal{N}} \overline{\mathcal{M}}$. Then for any $f \in \mathcal{L}$ with $\bar{f} \in \overline{\mathcal{M}} \overline{\mathcal{N}}$, there exists $h \in \mathcal{H}$ with $\bar{f}=\bar{h}$. By 3.7(a), we can choose $h$ to by $\uparrow \mathcal{K}$-maximal. So by 3.5, $f \in \mathcal{K} h \subseteq \mathcal{H}$. This shows $\mathcal{H}=\{f \in \mathcal{L}: \bar{f} \in \overline{\mathcal{M}} \overline{\mathcal{N}}\}$.

We need to prove that $\mathcal{H}=\mathcal{M N}=\mathcal{N} \mathcal{M}$. As the situation is symmetric in $\mathcal{M}$ and $\mathcal{N}$, it is enough to prove that $\mathcal{H}=\mathcal{M N}$. Since $\rho$ is a homomorphism, for any $m \in \mathcal{M}$ and $n \in \mathcal{N}$ with $(m, n) \in \boldsymbol{D}$, we have $\overline{m n}=\bar{m} \bar{n} \in \overline{\mathcal{M}} \overline{\mathcal{N}}$ and thus $m n \in \mathcal{H}$. Hence, $\mathcal{M N} \subseteq \mathcal{H}$. By 4.4, we have $\mathcal{H} \subseteq \mathcal{M N}$, so $\mathcal{H}=\mathcal{M N}$. Moreover, 4.4 shows that for every $f \in \mathcal{M N}$, there exists $m \in \mathcal{M}$ and $n \in \mathcal{N}$ such that $(m, n) \in \boldsymbol{D}, f=m n$, and $S_{f}=S_{(m, n)}$. So it only remains to prove that $S \cap(\mathcal{M N})=(S \cap \mathcal{M})(S \cap \mathcal{N})$. Clearly, $(S \cap \mathcal{M})(S \cap \mathcal{N}) \subseteq S \cap(\mathcal{M N})$. Let now $s \in S \cap(\mathcal{M N})$. By what we just said, there exists $m \in \mathcal{M}$ and $n \in \mathcal{N}$ with $(m, n) \in \boldsymbol{D}, s=m n$, and $S_{s}=S_{(m, n)}$. As $S_{s}=S$, it follows that $m, n \in G:=N_{\mathcal{L}}(S)$. By 2.5(a), $G$ is a subgroup of $\mathcal{L}$. Furthermore, property (L1) in the definition of a locality implies that $S$ is a Sylow 
$p$-subgroup of $G$. Note that $X:=G \cap \mathcal{M}$ and $Y:=G \cap \mathcal{N}$ are normal subgroups of $G$. Hence, $s=m n \in(X Y) \cap S=(X \cap S)(Y \cap S)=(\mathcal{M} \cap S)(\mathcal{N} \cap S)$ completing the proof.

\section{The Proof of Theorem 2}

Throughout, let $(\mathcal{L}, \Delta, S)$ be a locality with partial normal subgroups $\mathcal{N}_{1}, \ldots, \mathcal{N}_{l}$. We prove Theorem 2 in a series of lemmas.

5.1. Let $1 \leq k<l$ such that the products $\mathcal{N}_{1} \mathcal{N}_{2} \cdots \mathcal{N}_{k}$ and $\mathcal{N}_{k+1} \mathcal{N}_{k+2} \cdots \mathcal{N}_{l}$ are partial normal subgroups. Suppose furthermore that for any $f \in \mathcal{N}_{1} \cdots \mathcal{N}_{k}$ and any $g \in \mathcal{N}_{k+1} \cdots \mathcal{N}_{l}$ there exist $u=\left(n_{1}, \ldots, n_{k}\right), v=\left(n_{k+1}, \ldots, n_{l}\right) \in \boldsymbol{D}$ such that $n_{i} \in \mathcal{N}_{i}$ for $i=1, \ldots, l, f=\Pi(u), g=\Pi(v), S_{f}=S_{u}$ and $S_{g}=S_{v}$. Then

$$
\mathcal{N}_{1} \mathcal{N}_{2} \cdots \mathcal{N}_{l}=\left(\mathcal{N}_{1} \mathcal{N}_{2} \cdots \mathcal{N}_{k}\right)\left(\mathcal{N}_{k+1} \mathcal{N}_{k+2} \cdots \mathcal{N}_{l}\right)
$$

is a partial normal subgroup of $\mathcal{L}$, and for every $h \in \mathcal{N}_{1}, \ldots, \mathcal{N}_{l}$ there exists $w=\left(n_{1}, \ldots, n_{l}\right) \in \boldsymbol{D}$ such that $n_{i} \in \mathcal{N}_{i}$ for $i=1, \ldots, l, h=\Pi(w)$, and $S_{h}=S_{w}$.

Proof. By Theorem 1, $\left(\mathcal{N}_{1} \mathcal{N}_{2} \cdots \mathcal{N}_{k}\right)\left(\mathcal{N}_{k+1} \mathcal{N}_{k+2} \cdots \mathcal{N}_{l}\right)$ is a partial normal subgroup of $\mathcal{L}$. If $w=\left(n_{1}, \ldots, n_{l}\right) \in \boldsymbol{D}$ with $n_{i} \in \mathcal{N}_{i}$ for $i=1, \ldots, l$, then $u=$ $\left(n_{1}, \ldots, n_{k}\right), v=\left(n_{k+1}, \ldots, n_{l}\right) \in \boldsymbol{D}$, and

$$
\Pi(w)=\Pi(\Pi(u), \Pi(v)) \in\left(\mathcal{N}_{1} \mathcal{N}_{2} \cdots \mathcal{N}_{k}\right)\left(\mathcal{N}_{k+1} \mathcal{N}_{k+2} \cdots \mathcal{N}_{l}\right) .
$$

This proves that $\mathcal{N}_{1} \mathcal{N}_{2} \cdots \mathcal{N}_{l} \subseteq\left(\mathcal{N}_{1} \mathcal{N}_{2} \cdots \mathcal{N}_{k}\right)\left(\mathcal{N}_{k+1} \mathcal{N}_{k+2} \cdots \mathcal{N}_{l}\right)$. To prove the converse inclusion, let

$$
h \in\left(\mathcal{N}_{1} \mathcal{N}_{2} \cdots \mathcal{N}_{k}\right)\left(\mathcal{N}_{k+1} \mathcal{N}_{k+2} \cdots \mathcal{N}_{l}\right) .
$$

Then by Theorem 1 , there exist $f \in \mathcal{N}_{1} \cdots \mathcal{N}_{k}$ and $g \in \mathcal{N}_{k+1} \cdots \mathcal{N}_{l}$ such that $(f, g) \in \boldsymbol{D}, h=f g$, and $S_{h}=S_{(f, g)}$. By assumption, there exist $u=\left(n_{1}, \ldots, n_{k}\right)$ and $v=\left(n_{k+1}, \ldots, n_{l}\right) \in \boldsymbol{D}$ such that $n_{i} \in \mathcal{N}_{i}$ for $i=1, \ldots, l, f=\Pi(u), g=\Pi(v)$, $S_{f}=S_{u}$, and $S_{g}=S_{v}$. Then $S_{h}=S_{(f, g)}=S_{u \circ v}, u \circ v \in \boldsymbol{D}$ via $S_{h}$, and

$$
h=f g=\Pi(\Pi(u), \Pi(v))=\Pi(u \circ v) \in \mathcal{N}_{1} \mathcal{N}_{2} \cdots \mathcal{N}_{l},
$$

proving the assertion.

5.2. (a) The product $\mathcal{N}_{1} \mathcal{N}_{2} \cdots \mathcal{N}_{l}$ is a partial normal subgroup, and for every $f \in \mathcal{N}_{1} \mathcal{N}_{2} \cdots \mathcal{N}_{l}$ there exists $w=\left(n_{1}, \ldots, n_{l}\right) \in \boldsymbol{D}$ such that $n_{i} \in \mathcal{N}_{i}$ for $i=1, \ldots, l$, $f=\Pi(w)$, and $S_{f}=S_{w}$.

(b) For every $1 \leq k<$, we have

$$
\mathcal{N}_{1} \mathcal{N}_{2} \cdots \mathcal{N}_{l}=\left(\mathcal{N}_{1} \mathcal{N}_{2} \cdots \mathcal{N}_{k}\right)\left(\mathcal{N}_{k+1} \mathcal{N}_{k+2} \cdots \mathcal{N}_{l}\right)
$$


Proof. We prove this by induction on $l$. Clearly, the claim is true for $l=1$. Assume now $l>1$. Then there exists always $1 \leq k<l$. For any such $k$, it follows by induction (one time applied with $\mathcal{N}_{1}, \ldots, \mathcal{N}_{k}$ and one time applied with $\mathcal{N}_{k+1}, \ldots, \mathcal{N}_{l}$ in place of $\mathcal{N}_{1}, \ldots, \mathcal{N}_{l}$ ) that the hypothesis of 5.1 is fulfilled, so the assertion follows.

5.3. Let $\sigma \in S_{l}$ be a permutation. Then $\mathcal{N}_{1} \mathcal{N}_{2} \cdots \mathcal{N}_{l}=\mathcal{N}_{1 \sigma} \mathcal{N}_{2 \sigma} \cdots \mathcal{N}_{l \sigma}$.

Proof. We may assume that $\sigma=(i, i+1)$ for some $1 \leq i<l$, as $S_{l}$ is generated by transpositions of this form. Note that $\mathcal{N}_{1} \cdots \mathcal{N}_{i-1}, \mathcal{N}_{i} \mathcal{N}_{i+1}$ and $\mathcal{N}_{i+2} \cdots \mathcal{N}_{l}$ are partial normal subgroups by 5.2(a), where it is understood that $\mathcal{N}_{r} \cdots \mathcal{N}_{s}=\{\mathbf{1}\}$ if $r>s$. By Theorem 1 , we have $\mathcal{M N}=\mathcal{N} \mathcal{M}$ for any two partial normal subgroups. Using this fact and 5.2(b) repeatedly, we obtain

$$
\begin{aligned}
\mathcal{N}_{1} \mathcal{N}_{2} \cdots \mathcal{N}_{l} & =\left(\mathcal{N}_{1} \cdots \mathcal{N}_{i-1}\right)\left(\mathcal{N}_{i} \mathcal{N}_{i+1}\right)\left(\mathcal{N}_{i+2} \cdots \mathcal{N}_{l}\right) \\
& =\left(\mathcal{N}_{1} \cdots \mathcal{N}_{i-1}\right)\left(\mathcal{N}_{i+1} \mathcal{N}_{i}\right)\left(\mathcal{N}_{i+2} \cdots \mathcal{N}_{l}\right) \\
& =\mathcal{N}_{1 \sigma} \mathcal{N}_{2 \sigma} \cdots \mathcal{N}_{l \sigma}
\end{aligned}
$$

Proof of Theorem 2. It follows from 5.2(a) that $\mathcal{N}_{1} \cdots \mathcal{N}_{l}$ is a partial normal subgroup and that (c) holds. Property (a) is 5.2(b), and property (b) is 5.3.

\section{References}

[Aschbacher 2008] M. Aschbacher, "Normal subsystems of fusion systems", Proc. Lond. Math. Soc. (3) 97:1 (2008), 239-271. MR 2009e:20044 Zbl 1241.20026

[Aschbacher 2011] M. Aschbacher, The generalized Fitting subsystem of a fusion system, Memoirs of the American Mathematical Society 209:986, American Mathematical Society, Providence, RI, 2011. MR 2012f:20063 Zbl 1278.20020

[Broto et al. 2003] C. Broto, R. Levi, and B. Oliver, "Homotopy equivalences of $p$-completed classifying spaces of finite groups", Invent. Math. 151:3 (2003), 611-664. MR 2004c:55031 Zbl 1042.55008

[Broto et al. 2005] C. Broto, N. Castellana, J. Grodal, R. Levi, and B. Oliver, "Subgroup families controlling p-local finite groups", Proc. London Math. Soc. (3) 91:2 (2005), 325-354. MR 2007e:20111 Zbl 1090.20026

[Broto et al. 2007] C. Broto, N. Castellana, J. Grodal, R. Levi, and B. Oliver, "Extensions of $p$-local finite groups", Trans. Amer. Math. Soc. 359:8 (2007), 3791-3858. MR 2008i:55013 Zbl 1145.55013

[Chermak 2013] A. Chermak, "Fusion systems and localities", Acta Math. 211:1 (2013), 47-139. MR 3118305 Zbl 1295.20021

[Chermak 2015] A. Chermak, "Localities and projections", preprint, 2015. arXiv 1505.07786v2

[Henke 2015] E. Henke, "Subcentric linking systems", preprint, 2015. arXiv 1506.01458

[Oliver and Ventura 2007] B. Oliver and J. Ventura, "Extensions of linking systems with $p$-group kernel”, Math. Ann. 338:4 (2007), 983-1043. MR 2008k:55029 Zbl 1134.55011

Received June 2, 2015. Revised August 30, 2015. 
ELLEN HenKe

Institute of MATHEMATICS

UNIVERSITY OF ABERDEEN

FRASER Noble BUILDING

ABERDEEN

AB2 4 3UE

UNITED KINGDOM

ellen.henke@abdn.ac.uk 


\title{
PACIFIC JOURNAL OF MATHEMATICS
}

\author{
msp.org/pjm
}

Founded in 1951 by E. F. Beckenbach (1906-1982) and F. Wolf (1904-1989)

\section{EDITORS}

Don Blasius (Managing Editor)

Department of Mathematics

University of California

Los Angeles, CA 90095-1555

blasius@math.ucla.edu

\author{
Paul Balmer \\ Department of Mathematics \\ University of California \\ Los Angeles, CA 90095-1555 \\ balmer@math.ucla.edu \\ Robert Finn \\ Department of Mathematics \\ Stanford University \\ Stanford, CA 94305-2125 \\ finn@math.stanford.edu \\ Sorin Popa \\ Department of Mathematics \\ University of California \\ Los Angeles, CA 90095-1555 \\ popa@math.ucla.edu
}

\author{
Vyjayanthi Chari \\ Department of Mathematics \\ University of California \\ Riverside, CA 92521-0135 \\ chari@math.ucr.edu \\ Kefeng Liu \\ Department of Mathematics \\ University of California \\ Los Angeles, CA 90095-1555 \\ liu@math.ucla.edu \\ Jie Qing \\ Department of Mathematics \\ University of California \\ Santa Cruz, CA 95064 \\ qing@ cats.ucsc.edu
}

\section{PRODUCTION}

Silvio Levy, Scientific Editor, production@msp.org

\section{SUPPORTING INSTITUTIONS}

ACADEMIA SINICA, TAIPEI

CALIFORNIA INST. OF TECHNOLOGY

INST. DE MATEMÁTICA PURA E APLICADA

KEIO UNIVERSITY

MATH. SCIENCES RESEARCH INSTITUTE

NEW MEXICO STATE UNIV.

OREGON STATE UNIV.

\author{
STANFORD UNIVERSITY \\ UNIV. OF BRITISH COLUMBIA \\ UNIV. OF CALIFORNIA, BERKELEY \\ UNIV. OF CALIFORNIA, DAVIS \\ UNIV. OF CALIFORNIA, LOS ANGELES \\ UNIV. OF CALIFORNIA, RIVERSIDE \\ UNIV. OF CALIFORNIA, SAN DIEGO \\ UNIV. OF CALIF., SANTA BARBARA
}

\author{
Daryl Cooper \\ Department of Mathematics \\ University of California \\ Santa Barbara, CA 93106-3080 \\ cooper@math.ucsb.edu \\ Jiang-Hua Lu \\ Department of Mathematics \\ The University of Hong Kong \\ Pokfulam Rd., Hong Kong \\ jhlu@maths.hku.hk \\ Paul Yang \\ Department of Mathematics \\ Princeton University \\ Princeton NJ 08544-1000 \\ yang@math.princeton.edu
}

These supporting institutions contribute to the cost of publication of this Journal, but they are not owners or publishers and have no responsibility for its contents or policies.

See inside back cover or msp.org/pjm for submission instructions.

The subscription price for 2015 is US \$420/year for the electronic version, and \$570/year for print and electronic.

Subscriptions, requests for back issues and changes of subscribers address should be sent to Pacific Journal of Mathematics, P.O. Box 4163, Berkeley, CA 94704-0163, U.S.A. The Pacific Journal of Mathematics is indexed by Mathematical Reviews, Zentralblatt MATH, PASCAL CNRS Index, Referativnyi Zhurnal, Current Mathematical Publications and Web of Knowledge (Science Citation Index).

The Pacific Journal of Mathematics (ISSN 0030-8730) at the University of California, c/o Department of Mathematics, 798 Evans Hall \#3840, Berkeley, CA 94720-3840, is published twelve times a year. Periodical rate postage paid at Berkeley, CA 94704, and additional mailing offices. POSTMASTER: send address changes to Pacific Journal of Mathematics, P.O. Box 4163, Berkeley, CA 94704-0163.

PJM peer review and production are managed by EditFLOW ${ }^{\circledR}$ from Mathematical Sciences Publishers.

\section{PUBLISHED BY}

\section{mathematical sciences publishers \\ nonprofit scientific publishing}

http://msp.org/

(C) 2015 Mathematical Sciences Publishers 


\title{
PACIFIC JOURNAL OF MATHEMATICS
}

\author{
Volume 279 No. 1-2 December 2015
}

In memoriam: Robert Steinberg

Robert Steinberg (1922-2014): In memoriam V. S. VARADARAJAN

Cellularity of certain quantum endomorphism algebras

HENNING H. ANDERSEN, GUSTAV I. LEHRER and RUIBIN ZHANG

Lower bounds for essential dimensions in characteristic 2 via orthogonal representations ANTONIO BABIC and VLADIMIR CHERNOUSOV

Cocharacter-closure and spherical buildings

Michael Bate, Sebastian Herpel, Benjamin Martin and Gerhard RöHrLe

Embedding functor for classical groups and Brauer-Manin obstruction

Eva Bayer-Fluckiger, Ting-Yu LeE and Raman Parimala

On maximal tori of algebraic groups of type $G_{2}$

Constantin Beli, PhilipPe Gille and Ting-Yu LeE

On extensions of algebraic groups with finite quotient

MICHEL BRION

Essential dimension and error-correcting codes

SHANE CERNELE and ZiNOVy REICHSTEIN

Notes on the structure constants of Hecke algebras of induced representations of finite Chevalley groups

Charles W. CuRTis

Complements on disconnected reductive groups

FRANÇOIS DIGNE and JEAN MICHEL

Extending Hecke endomorphism algebras

Jie Du, Brian J. Parshall and LeOnard L. SCOTT

Products of partial normal subgroups

ELLEN HENKE

Lusztig induction and $\ell$-blocks of finite reductive groups

RADHA KESSAR and GUNTER MALLE

Free resolutions of some Schubert singularities

Manoj Kummini, Venkatramani Lakshmibai, Pramathanath Sastry and C. S. Seshadri

Free resolutions of some Schubert singularities in the Lagrangian Grassmannian

VenKatramani LAKSHMibai and ReUVEN HODGES

Distinguished unipotent elements and multiplicity-free subgroups of simple algebraic groups

Martin W. Liebeck, Gary M. Seitz and Donna M. Testerman

Action of longest element on a Hecke algebra cell module

GEORGE LUSZTIG

Generic stabilisers for actions of reductive groups

BENJAMIN MARTIN

On the equations defining affine algebraic groups

VLADIMIR L. POPOV

Smooth representations and Hecke modules in characteristic $p$

PETER SCHNEIDER

On CRDAHA and finite general linear and unitary groups

BHAMA SRINIVASAN

Weil representations of finite general linear groups and finite special linear groups PHAM HUU TIEP

The pro- $p$ Iwahori Hecke algebra of a reductive $p$-adic group, $\mathrm{V}$ (parabolic induction) MARIE-FRANCE VIGNÉRAS

Acknowledgement 